\title{
Nasal mucociliary clearance in prolonged tracheostomy patients -A prospective case-control study.
}

\author{
Prerit Rao ${ }^{1}$, ROHIT SINGH${ }^{1}$, Dipak Nayak ${ }^{1}$, and ramaswamy Balakrishnan ${ }^{1}$ \\ ${ }^{1}$ Kasturba Medical College Manipal
}

October 16, 2020

\begin{abstract}
Background: Prolonged tracheostomy bypasses the upper airways leading to absence or reduction of nasal airflow. This altered nasal physiology not only impairs olfaction but also may cause mucociliary dysfunction and consequent nasal crusting and rhinosinusitis. Objective: To objectively evaluate the extent of nasal mucociliary impairment in patients with prolonged duration of tracheostomy. Design and Setting: This is a prospective case-control study done in a tertiary care center wherein the nasal mucocilary function was assessed by saccharin test in patients who were tracheostomized for a period of more than 4 weeks and the saccharin transit time (time taken to appreciate the sweet taste after endoscopic insertion of saccharin pellet on anterior end of inferior turbinate) was compared by the same test in age and sex matched healthy individuals. Main outcome measures: Clinical data, indication of tracheostomy and duration of tracheostomy were assessed for their impact on the nasal mucociliary clearance using saccharin transit time in the two groups which was then statistically analyzed using student t-test. Results: Mean saccharin transit time in 30 patients with prolonged tracheostomy was 934.97 seconds with a standard deviation of 75.95 seconds whereas in 30 controls, the meantime was 447.4 seconds with a standard deviation of 63.22 seconds, which was statistically significant $(\mathrm{p}<0.001)$ Conclusion: Prolonged duration of tracheostomy leads to impaired nasal mucociliary clearance, which in turn could cause chronic rhinosinusitis.
\end{abstract}

\section{ABSTRACT: \\ Background:}

Prolonged tracheostomy bypasses the upper airways leading to absence or reduction of nasal airflow. This altered nasal physiology not only impairs olfaction but also may cause mucociliary dysfunction and consequent nasal crusting and rhinosinusitis.

\section{Objective:}

To objectively evaluate the extent of nasal mucociliary impairment in patients with prolonged duration of tracheostomy.

\section{Design and Setting:}

This is a prospective case-control study done in a tertiary care center wherein the nasal mucocilary function was assessed by saccharin test in patients who were tracheostomized for a period of more than 4 weeks and the saccharin transit time (time taken to appreciate the sweet taste after endoscopic insertion of saccharin pellet on anterior end of inferior turbinate) was compared by the same test in age and sex matched healthy individuals.

Main outcome measures: Clinical data, indication of tracheostomy and duration of tracheostomy were assessed for their impact on the nasal mucociliary clearance using saccharin transit time in the two groups which was then statistically analyzed using student t-test. 


\section{Results:}

Mean saccharin transit time in 30 patients with prolonged tracheostomy was 934.97 seconds with a standard deviation of 75.95 seconds whereas in 30 controls, the meantime was 447.4 seconds with a standard deviation of 63.22 seconds, which was statistically significant $(\mathrm{p}<0.001)$

\section{Conclusion:}

Prolonged duration of tracheostomy leads to impaired nasal mucociliary clearance, which in turn could cause chronic rhinosinusitis.

\section{Key words: Nasal mucociliary clearance, saccharin test, prolonged tracheostomy}

\section{Key Points:}

1. Multiple factors determine normal nasal mucociliary clearance hence there is a lot of variation in the normal mucociliary clearance based on different ethnicity, gender and location.

2. Effects of prolonged tracheostomy altering the airflow and leading to mucociliary dysfunction, has not been extensively researched.

3. Saccharin test is a quick, reliable and economical test for evaluation of nasal mucociliary clearance time.

4. Mucociliary dysfunction worsens as duration of tracheostomy increases .

5. With the improved survival of prolonged tracheostomy patients, they need to be counselled and rehabilitated accordingly.

\section{Introduction:}

With the advances made in the field of rhinology in the last two decades, several studies have highlighted the functions of nasal mucocilia and its pivotal role in maintaining the nasal physiology. The focus of attention is now drawn to identify the factors which alter the nasal mucociliary clearance, thereby affecting the nasal functions.

Tracheostomy is a life-saving procedure that bypasses the upper airway causing absence or reduced nasal airflow. Hypothetically this altered nasal airflow in patients with prolonged tracheostomy could lead to nasal mucociliary dysfunction. There are very few studies in the medical literature that evaluates the effects of prolonged tracheostomy on nasal physiology ${ }^{[1]}$. Hence this study was done to assess the impact of prolonged tracheostomy on nasal mucociliary clearance.

\section{Methods}

Ethical Considerations:

The institutional ethics committee approved the protocol for the study, and we obtained clearance to conduct a prospective study.

\section{Study Design and Patients}

This prospective case-control study was done to objectively analyze the degree of nasal mucociliary impairment in 30 patients with prolonged tracheostomy when compared with that in age and sex matched 30 healthy individuals. This study was conducted in the Department of Otorhinolaryngology in a tertiary care center for a period of 2 years after obtaining clearance from the institutional ethics committee. The patients who were between the age of 20-60 years with no history of pre-existing ciliary disorders or naso-sinus 
pathology and who were tracheostomized for at least 4 weeks were recruited as cases. Similarly, healthy volunteers aged between 20-60 years with no known naso-sinus pathology were recruited as controls.

All subjects enrolled underwent the Saccharin test after taking an informed written consent in which a standardized $1 \mathrm{~mm}$ saccharin pellet was kept on the anterior end of inferior turbinate of one side under direct visualization with a nasal endoscope in supine position. Patients remained in the same position and were told to report as soon as they appreciated sweet taste in their throat. During the test the subjects were asked to quietly inhale and exhale and not to sniff, sneeze, eat, drink or swallow frequently. Time taken for the perception of sweet sensation in the throat was measured as saccharin transit time (STT).

All the parameters were then entered in a master chart, and statistical analysis of data was done using Microsoft Excel 2019 software. The Categorical variable was summarized by frequency and percentile, whereas the continuous variable was summarized by the mean and standard deviation. 'Independent sample t-test' was used to compare the meantime across two groups. The primary outcome of the study was measured as the alteration in nasal mucociliary clearance time by the saccharin test.

\section{Results:}

Both the case and control groups had 30 subjects included in this study. The sex ratio in the case group was 20:10 and in the control group was 18:12. The mean age of the subjects in the case group was 45.33 years with a standard deviation (SD) of \pm 11.8 years and in the control group the mean age was 39 years with a SD of \pm 12.15 years. Thus, the age and sex were well matched in the two groups. Most of the patients in both the groups were in the age group of 50-60 years (14/30 in the case group and 9/30 in the control group). In the case group, the minimum duration of tracheostomy at the time of the test was 28 days, and the maximum duration was 742 days and the most common indication for tracheostomy was stridor and the most common diagnosis was malignancy of upper aerodigestive tract. Nasal mucociliary clearance time in cases group ranged from 752 seconds to 1062 seconds with a mean time of 934.9 seconds (SD \pm 75.94 seconds) whereas, in the control group, nasal mucociliary clearance time ranged from 346 seconds to 576 seconds with a mean of 447.4 (SD \pm 63.21 seconds) as shown in chart 1. Another finding noted was that the impairment in nasal mucociliary clearance was directly proportional to the duration of tracheostomy (Chart 2). These results of nasal mucociliary clearance time in 2 groups were analyzed using the 'Independent sample t-test' to determine the p-value which was $<0.001$ and which was statistically significant indicating a worsening of nasal mucociliary clearance in tracheostomy patients.

\section{Discussion:}

In 1981, Puchelle et al. showed that the saccharin test could be used as a useful screening test in nasal mucociliary clearance. ${ }^{[2]}$ Various authors have also shown that saccharin test is advantageous over other modalities of investigation. Hence to assess the impact of tracheostomy on nasal mucociliary clearance, a saccharin test was performed.

In 1995, Kleinschmidt et al. used the saccharin test and showed a saccharin transit time (STT) of 624 seconds in healthy subjects while the present study showed a mean STT of $447.4 \pm 63.2$ seconds in control group. ${ }^{[3]}$

Similar studies were done by Yergin et al., Stanley et al. showed a mean STT of 432 seconds, $702 \pm 228$ seconds, respectively, in healthy subjects. Such variations in average mucociliary clearance time are attributed to various factors such as habits, habitat, climate, facial configuration, and comorbidities ${ }^{[4,5,6]}$. A study done by Golhar et al. on 150 subjects from India revealed a mean nasal mucociliary clearance time of 430 seconds (range: 320- 700 seconds) which was consistent with the results of the present study. Golhar further stressed that the saccharin test could be used as a valid screening test, not only for the diagnosis of nasal mucociliary impairment but also for the prognosis of various diseases of nasal and lower respiratory tracts ${ }^{[7]}$. Karaoglu 
D et al. conducted a study in which they studied the long-term effects of laryngectomy on nasal functions and found that mean mucociliary clearance time for laryngectomized patients was 1017.14 seconds whereas in control group it was 662.5 seconds suggesting that physiological alteration in the nose due to bypass of nasal airway, leads to reduced ciliary clearance and impaired olfaction which can affect patients quality of life and safety. ${ }^{[8]}$

In our study, mean nasal mucociliary clearance time (NMCT) was $934.97 \pm 75.9$ seconds, these results were similar to study done by El-Sharnouby et al. who conducted a study on 31 patients and found that the mean saccharine test time recorded 2 months following tracheostomy was $732.6 \pm 323.4$ seconds and at 6 months postoperatively was $1615 \pm 1042$ seconds and was statistically significant. ${ }^{[9]}$ However, study done by Yadav SP et al. on 30 tracheostomized patients showed nasal mucociliary clearance time of $444 \pm 22.8$ seconds when saccharin test was done at 3 weeks following tracheostomy and NMCT further reduced to $371.4 \pm 32.4$ seconds in patients who were tracheostomized for more than 3 weeks, suggesting that, following tracheostomy, there is a significant decrease in nasal mucociliary clearance time, particularly after 3 weeks postoperatively. ${ }^{[10]}$

A study was done by Tsikoudas A et al. in 2011 on 10 subjects in cases and control arms respectively, where the author used SNOT-22, and Rhinogram nasal symptoms score questionnaires and found that tracheostomy group had a median Rhinogram score of 13.5 vs. 2 for the control arm $(\mathrm{p}=0.02)$, and similarly SNOT-22 scores for tracheostomized patients and controls were 39 and 29 respectively $(\mathrm{p}=0.205)$, thereby observing that the tracheostomy group had more nasal symptoms due to worsening of mucociliary clearance, which supports the hypothesis of this present study and author concluded by stressing that this impairment in nasal mucociliary clearance following tracheostomy should be addressed in follow up as altered nasal function can predispose patients to sinonasal diseases and a reduction in olfaction causing poor identification of smell of food leading to a poor quality of life and could become potentially dangerous in situations like gas leak. ${ }^{[1]}$

Smaller sample size was one of the limitations of this study hence further studies with larger sample size and follow-up can be done to better understand these changes in nasal mucocilia and whether these changes are reversible following decannulation, additionally we can also determine whether techniques like polite yawning etc. can be used to improve nasal mucociliary physiology and olfaction in these tracheotomised patients.

\section{Conclusion:}

Nasal mucociliary clearance is an important primary defense mechanism in the nose and paranasal sinus, which is dependent on various factors and can be impaired due to environmental factors, patient factors, and disease factors. As tracheostomy bypasses the upper airway, it also leads to altered nasal physiology such as changes in nasal airflow, nasal cycle, and most importantly, alteration of nasal mucociliary clearance. In our study, we found out that there is a prolonged saccharin test implying a reduction of nasal mucociliary clearance following tracheostomy when compared with age and sex-matched healthy individuals. This finding is of clinical significance, as patients need to be counselled regarding the changes in nasal function such as altered olfaction and increased risk of nasal crusting and sino-nasal diseases.

\section{Acknowledgment: Nil}

\section{Funding: Nil}

\section{Conflict of interest: Nil}

Data availability statement: The data supporting the findings of this study are available from the corresponding author upon reasonable request.

\section{References:}


1. Tsikoudas A, Barnes ML, White P. The impact of tracheostomy on the nose. Eur Arch Otorhinolaryngol. 2011; 268(7): 1005-8.

2. Puchelle EF, Aug F, Pham QT, Bertrand A. Comparison of three methods for measuring nasal mucociliary clearance in man. Acta otolaryngologica. 1981; 91(1-6): 297-303.

3. Kleinschmidt EG, Witt G. Evaluation of nasal mucociliary clearance with a modified saccharin test. Laryngo-rhino-otologie. 1995; 74(5): 286-8.

4. Yergin BM, Saketkhoo KI, Michaelson ED, Serafini SM, Kovitz KE, Sackner MA. A roentgenographic method for measuring nasal mucous velocity. Journal of Applied Physiology. 1978; 44(6): 964-8.

5. Stanley P, MacWilliam L, Greenstone M, Mackay I, Cole P. Efficacy of a saccharin test for screening to detect abnormal mucociliary clearance. British Journal of Diseases of the chest. 1984; 78: 62-5.

6. Canciani M, Barlocco EG, Mastella G, De Santi MM, Gardi C, Lungarella G. The saccharin method for testing mucociliary function in patients suspected of having primary ciliary dyskinesia. Pediatric pulmonology. 1988; 5(4): 210-4.

7. Golhar S. Nasal mucus clearance. The Journal of Laryngology \& Otology. 1986; 100 (5): 533-8.

8. Karaoglu D, Kocyigit M, Ortekin SG, Adali MK. Late-term Effects of Surgery on Nasal Functions in Patients who Underwent Total Laryngectomy Surgery. International archives of otorhinolaryngology. 2017; 21(03): 270-5.

9. El-Sharnouby M, Khalil Y, El-Shafy IA, Khalil M. Evaluation of nasal mucosal changes of tracheastomized patients. Menoufia Med J. 2018; 31: 254-61.

10. Yadav SP, Janab KS, Singh J, Ranga RK. Nasal mucociliary clearance in patients with tracheostomy. Indian J Otolaryngol Head Neck Surg. 2003; 55(2); 73-75.

11. Tsikoudas A, Barnes ML, White P. The impact of tracheostomy on the nose. Eur Arch Otorhinolaryngol. 2011; 268(7): 1005-8.

\section{Figure legends:}

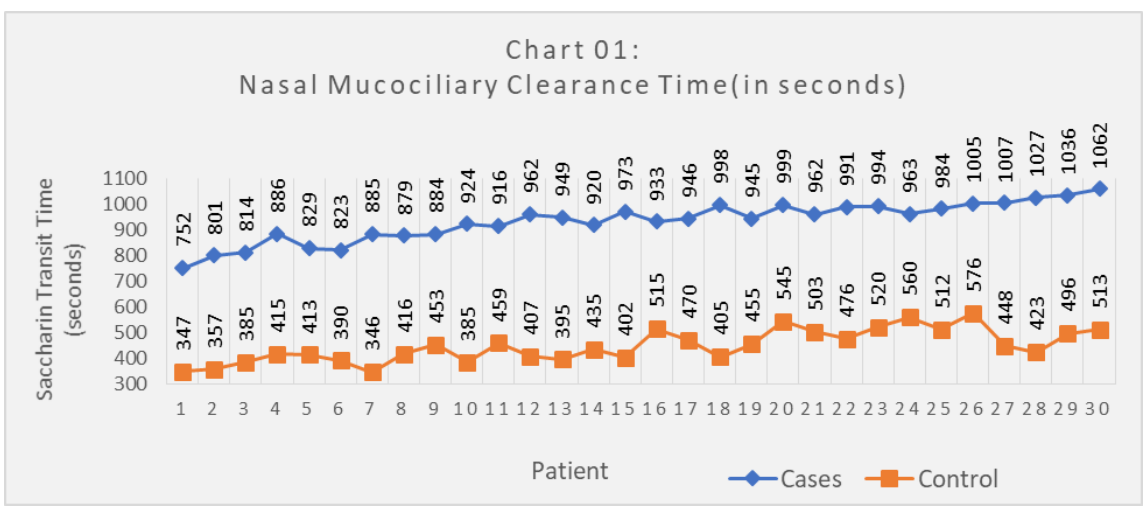

Chart 01: Nasal mucociliary clearance time (in seconds)

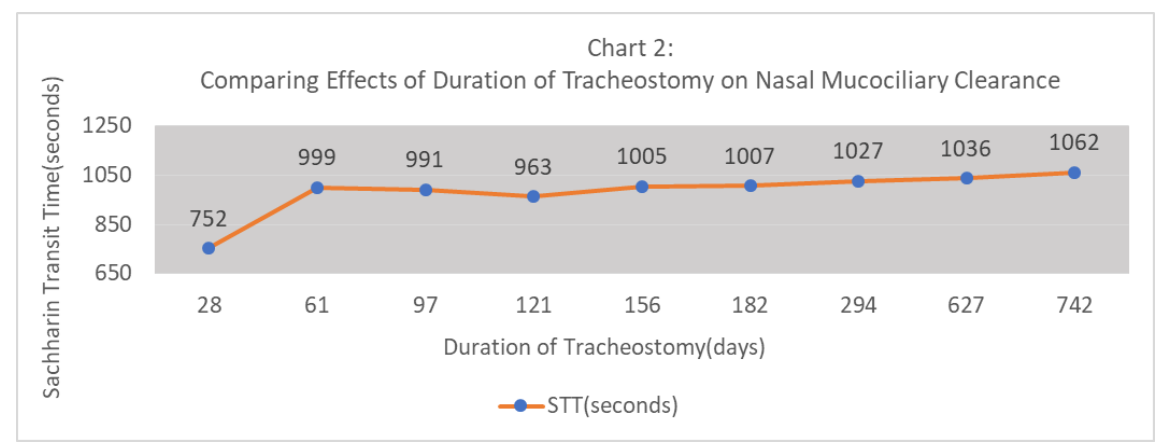


Chart 02: Comparing effects of duration of tracheostomy on nasal mucociliary clearance 